\title{
Clostridium septicum Aortitis in a Patient with Colon Carcinoma
}

Liechti, M E ; et al

DOI: https://doi.org/10.1007/s10096-003-1009-0

Posted at the Zurich Open Repository and Archive, University of Zurich ZORA URL: https://doi.org/10.5167/uzh-156543

Journal Article

Published Version

Originally published at:

Liechti, M E; et al (2003). Clostridium septicum Aortitis in a Patient with Colon Carcinoma. European Journal of Clinical Microbiology Infectious Diseases, 22(10):632-634.

DOI: https://doi.org/10.1007/s10096-003-1009-0 


\section{Clostridium septicum Aortitis in a Patient with Colon Carcinoma}

Published online: 25 September 2003

(C) Springer-Verlag 2003

Clostridium septicum is an anaerobic, spore-forming and toxin-producing gram-positive rod with peritrichous flagella. Septicemia due to this organism is severe and has an overall mortality rate of $33-64 \%[1,2]$. Clostridium septicum is a known cause of gas gangrene. However, the clinical importance of Clostridium septicum septicemia lies in its association with gastrointestinal or hematologic malignancy, with the most common site being adenocarcinoma of the right colon [3]. Vascular infection with Clostridium septicum has rarely been reported and might occur when circulating bacteria infect an atheromatous lesion during bacteremia [4, 5]. Here, we describe a patient with occult adenocarcinoma of the colon who presented with early-stage clostridial aortitis that progressed to an infected aneurysm.

A 55-year-old man was admitted to hospital with abdominal pain and fever in August 2002. He had a 3year-history of primary biliary cirrhosis, and 20 years previously a seminoma had been treated with radiotherapy. On examination, he had a temperature of $38^{\circ} \mathrm{C}$. The abdomen was tender with no palpable masses.

M. E. Liechti · B. Caduff

Department of Medicine,

Limmattal Hospital,

8952 Zurich, Switzerland

O. Schöb

Department of Surgery,

Limmattal Hospital,

8952 Zurich, Switzerland

G. M. Kacl

Department of Radiology and Nuclear Medicine,

Limmattal Hospital,

8952 Zurich, Switzerland

M. E. Liechti (

Department of Internal Medicine,

University Hospital Zurich,

Rämistrasse 100, 8091 Zurich, Switzerland

e-mail: matthias.liechti@usz.ch

Tel.: +41-1-2551111

Fax: +41-1-2554445
Blood tests showed a raised leukocyte count of $13 \times 10^{3} / \mathrm{mm}^{3}$ with $75 \%$ neutrophils and $39 \%$ band forms, an elevated C-reactive protein level of $194 \mathrm{mg} / \mathrm{l}$, and raised alkaline phosphatase of $235 \mathrm{U} / 1$ and gamma glutamyltransferase of $176 \mathrm{U} / \mathrm{l}$. The hemoglobin concentration was $6.1 \mathrm{~g} / \mathrm{dl}$. Blood cultures grew an anaerobic rod identified as Clostridium septicum. Computed tomography (CT) showed an infrarenal periaortic mass (Fig. 1). The differential diagnosis included infectious aortitis, retroperitoneal lymphadenopathy, fibrosis, or neoplasm. Intravenous therapy with amoxicillin clavulanate and metronidazole was initiated, and the patient's condition improved rapidly.

Colonoscopy and CT revealed a large adenocarcinoma of the transverse colon. After 2 weeks of antibiotic therapy the transverse colon was surgically resected. Histology confirmed the presence of an ulcerating adenocarcinoma without lymph node involvement. An intraoperative periaortic lymph node biopsy showed

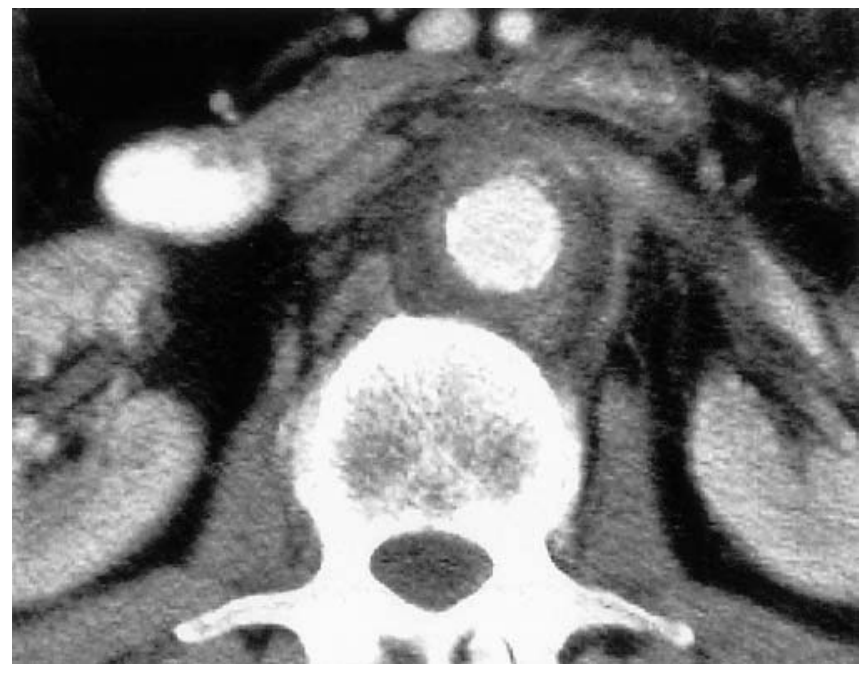

Fig. 1 Abdominal computed tomography scan showing circumferential soft-tissue thickening around the aorta (early-stage aortitis) at hospital admission 


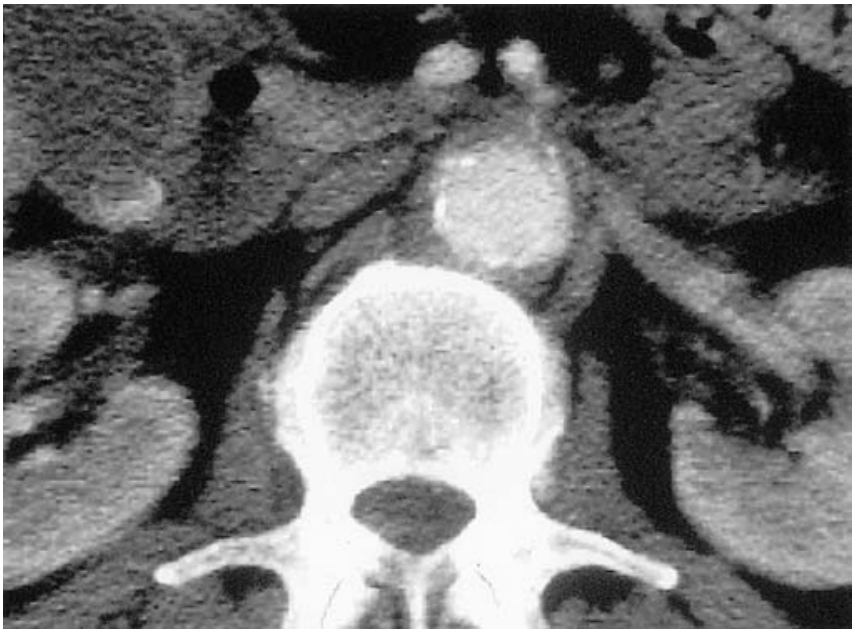

Fig. 2 Complete regression of the periaortic tissue thickening after 1 month of antibiotic treatment

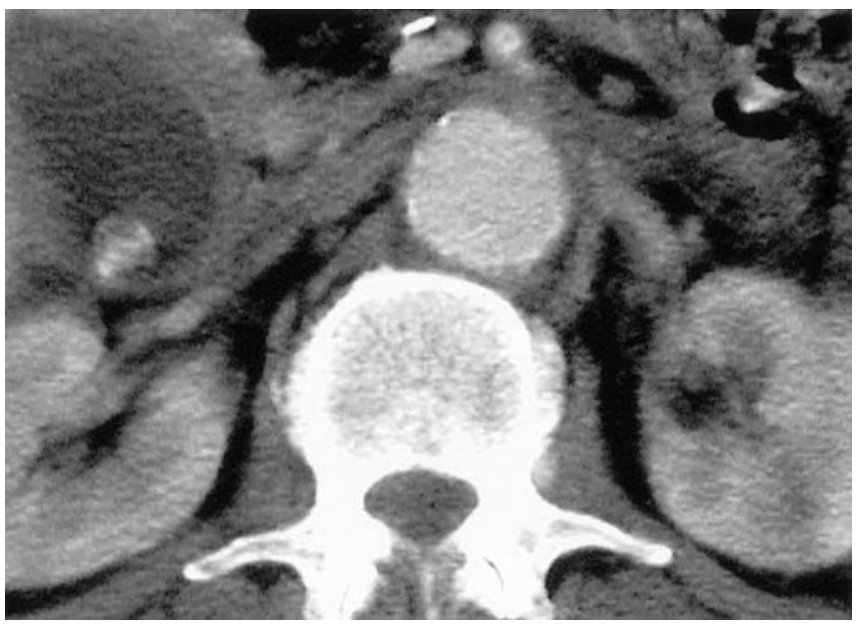

Fig. 3 Expansion of the aorta indicating progressing aortitis 3 months after admission

chronic inflammation but no bacteria or neoplasia. Therapy with amoxicillin/clavulanate and metronidazole was continued for a total of 4 weeks followed by levofloxacin for 3 weeks. A CT scan performed 4 weeks after initiation of antimicrobial therapy showed regression of the periaortic swelling (Fig. 2), but 3 months after admission, the patient again complained of increasing abdominal pain. There was no fever, but his leukocyte count and C-reactive protein level were elevated, and blood cultures grew Clostridium septicum. Computed tomography showed an increase in the size of the periaortic mass, expansion of the infrarenal aorta (up to $3 \mathrm{~cm}$ ), and an anterior disruption of the aortic mural calcification (Fig. 3).

On surgical exploration, a saccular aneurysm of the inflamed aorta was found close to the renal arteries. Aortic graft placement was not performed, however, because the necessary clamping of the renal and mesen- teric arteries was considered of high risk at that time, given the patient's reduced general condition. Instead, antibiotic therapy with levofloxacin was reinstated. Nevertheless, in the following weeks the patient's condition worsened. The patient refused further treatment and died 5 months after hospital admission from uncontrolled sepsis.

At present, 18 cases of Clostridium septicum aortic or iliac infections have been reported in the literature $[4,5$, $6,7,8,9]$. These cases were diagnosed during late-stage disease or at autopsy with infected aneurysms and gas in the surrounding tissues [4] or in connection with a dissecting or ruptured aneurysm [6]. All patients were older than 60 years (mean age, 73.6 years). Adenocarcinoma or polyps were found in the colon of 13 patients. Hematologic malignancy was present in one patient. Most of the patients had diabetes and severe atherosclerosis. Atherosclerotic lesions appear to facilitate hematogenous seeding of the arterial wall during bacteremia from the distant focus. Eleven patients died, most of them soon after diagnosis of the aortic infection. All eight patients who survived were treated with a combination of surgery and antibiotic therapy.

The patient we describe here was distinct from the patients reported previously in that the diagnosis of aortitis was made at an early stage in a comparatively young patient and prompt antibiotic treatment led to rapid clinical improvement and regression of the aortitis on CT. Initially, antibiotic treatment was considered sufficient and graft replacement was not performed. However, a false aneurysm developed, despite prolonged antibiotic treatment, and Clostridium septicum septicemia recurred, leading to death.

A periaortic soft tissue mass, increased contrast enhancement, and a normal-sized aorta, as observed in our patient, are the characteristic findings of early aortic infection on CT [10]. Rapid disease progression with expansion of the aortic lumen, formation of saccular aneurysms, and aortic rupture has been documented previously [5]. The present case illustrates that once Clostridium septicum becomes established in arteriosclerotic lesions, it cannot be eradicated with antibiotic therapy alone. Infection of the aorta should therefore be treated by both debridement of the infected segment, with proper reconstruction, and indefinite antibiotic therapy. In addition, the identification of Clostridium septicum in blood cultures should always prompt a search for associated occult malignancy.

\section{References}

1. Pelletier JPR, Plumbley JA, Rouse EA, Cina SJ (2000) The role of Clostridium septicum in paraneoplastic sepsis. Arch Pathol Lab Med 124:353-356

2. Pelfrey TM, Turk RP, Peoples JB, Elliott DW (1984) Surgical aspects of Clostridium septicum septicemia. Arch Surg 119:546-550

3. Alpern RJ, Dowell VR (1969) Clostridium septicum infections and malignancy. JAMA 209:385-388 
4. Morrison RC, DiMuzio PJ, Kahn M, Carabasi RA, Bailey W, Edie RN (2001) Clostridial mycotic aneurysm of the thoracoabdominal aorta. Vasc Surg 35:303-310

5. Messa CA, Kulkarni M, Arous E (1995) Double clostridial mycotic aneurysms of the aorta. Cardiovasc Surg 3:687-692

6. Upchurch GR, Clair DG, Whittemore AD, Mannick JA (1995) Clostridium septicum bacteremia associated with aortic graft infection. J Vasc Surg 22:493-495

7. Sailors DM, Eidt JF, Gagne PJ, Barnes RW, Barone GW, McFarland DR (1996) Primary Clostridium septicum aortitis: a rare cause of necrotizing suprarenal aortic infection-a case report and review of the literature. J Vasc Surg 23:714-718

8. Munshi IA, Rhee SW, Pane T, Granowitz E (2002) Clostridium septicum mycotic aortic aneurysm. Am J Surg 184:54-55

9. Zenati MA, Bonanomi G, Kostov D, Lee R (2002) Fulminant Clostridium septicum aortitis. Circulation 105:1871

10. Sueyoshi E, Sakamoto I, Kawahara Y, Hayashi K (1998) Infected abdominal aortic aneurysm: early CT findings. Abdom Imaging 23:645-648 